\title{
Des Images, des nœuds et des toiles
}

Pierres sacrées et ignames à Nyamikum (district de maprik, East Sepik province, Papouasie-Nouvelle Guinée)

Images, nodes and networks. The sacred stones of the yams of Nyamikum

(district of maprik, East Sepik province, apua New Guinea)

\section{Ludovic Coupaye}

\section{(2) OpenEdition}

Journals

Édition électronique

URL : https://journals.openedition.org/tc/6424

DOI : $10.4000 /$ tc. 6424

ISBN : $1952-420 X$

ISSN : 1952-420X

Éditeur

Éditions de l'EHESS

\section{Édition imprimée}

Date de publication : 15 juin 2012

Pagination : 142-159

ISBN : 2-7351-1512-7

ISSN : 0248-6016

Référence électronique

Ludovic Coupaye, «Des Images, des nœuds et des toiles », Techniques \& Culture [En ligne], 58 | 2012, mis en ligne le 30 octobre 2012, consulté le 29 septembre 2022. URL : http://journals.openedition.org/ tc/6424; DOI : https://doi.org/10.4000/tc.6424 


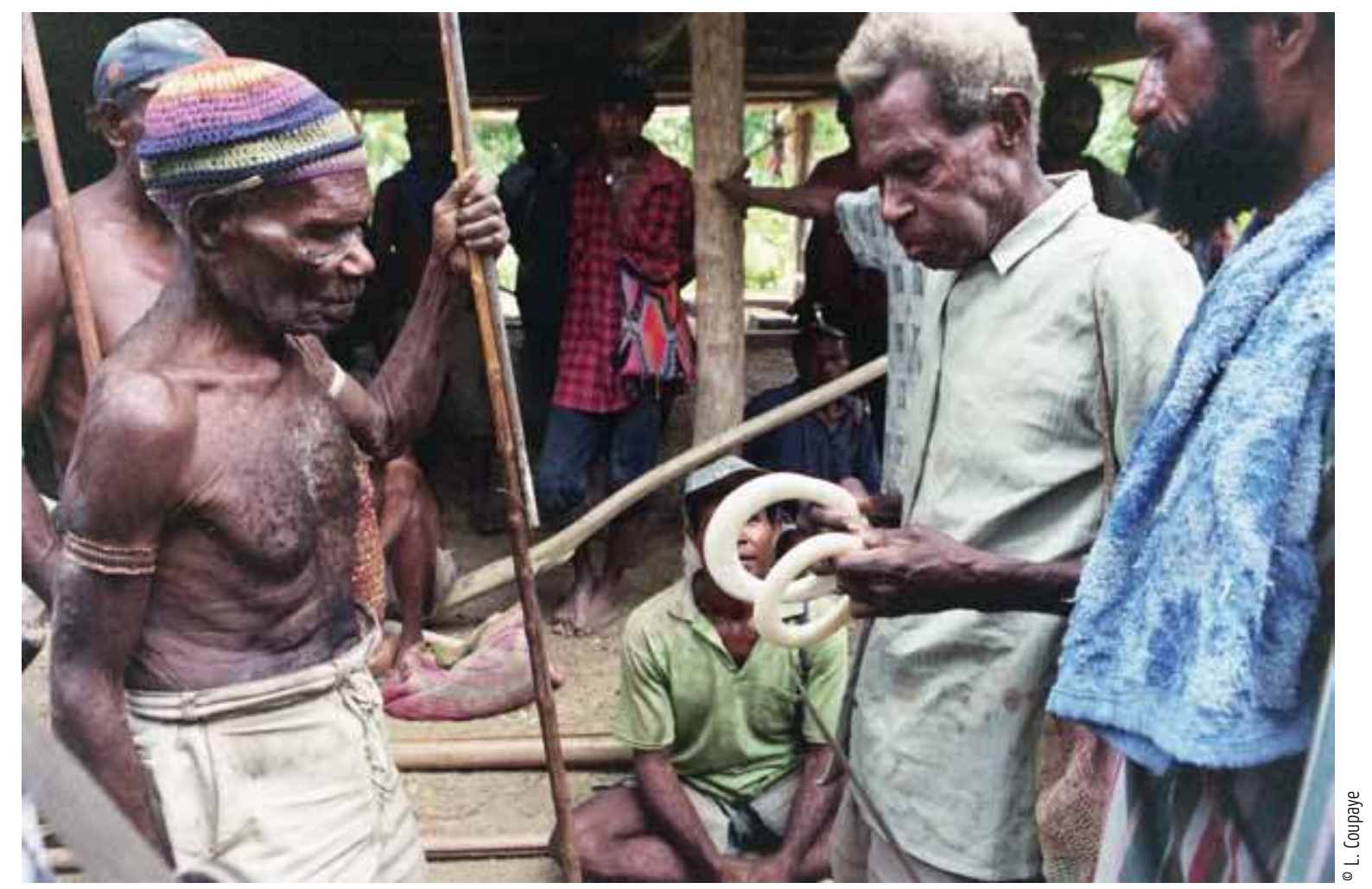




\section{DES IMAGES, DES NCEUDS ET DES TOILES}

\section{Pierres sacrées et ignames à Nyamikum (district de maprik, East Sepik province, Papouasie-Nouvelle-Guinée)}

Le caractère irremplaçable des objets (ici au sens restreint d'artefacts ou d'objets matériels) se manifeste en archéologie et anthropologie, sous deux formes principales. L'une, peut être qualifiée d'emic, c'est-à-dire irremplaçable du point de vue des groupes humains, l'autre, etic, pour les chercheurs eux-mêmes, comme focale et comme moyen de montrer ce que d'autres approches ne peuvent montrer seules, ou de montrer les liens entre des sujets généralement traités de manière séparée (Lemonnier 2012, Coupaye 2012).

Afin d'illustrer cette double approche, je prendrais pour exemple le cas des grandes ignames de la population Abelam, résidant aujourd'hui sur le versant sud des Prince Alexander Range, dans l'East Sepik Province, en Papouasie-Nouvelle-Guinée. Entrés dans l'anthropologie depuis leur rencontre avec Richard Thurnwald (1914), les Abelam ont été ethnographiés juste avant la guerre par Phyllis Kaberry (1941). Mais c'est surtout par les recherches d'Anthony Forge $(1965,1973)$ que les Abelam acquirent une position privilégiée dans la littérature anthropologique en raison de leurs cycles initiatiques complexes associés à une riche production visuelle que l'on trouve dans les collections de la plupart des musées euro-américains (cf. Smidt \& McGuigan 1994).

Si besoin était, il suffirait de suivre la généalogie des études de Forge pour voir le caractère aujourd'hui irremplaçable des œuvres Abelam dans une histoire de l'anthropologie des arts d'Océanie qui se poursuit avec Gell (2008 [1998]), Morphy (2005), Küchler (1992) ainsi que Hauser-Schäublin (1989a), Kaufmann (1993, 2005) et Revolon (2007), tous ayant contribué à installer ce domaine au cœur de l'anthropologie contemporaine de la culture matérielle. 
sont richement décorées d'éléments - également utilisés pour les décorations coutumières des initiés (Hauser-Schäublin 1995), et dont les formes évoquent celles des anciennes figures cérémonielles (Heermann 1983) - et exposées lors d'une cérémonie, appelée à Nyamikum, Waapi Saaki (« Alignement des grandes ignames »- Figure 2 et 3). Elles y sont évaluées par les habitants du village, ainsi que par des délégations de visiteurs venus d'autres villages. Cette cérémo-

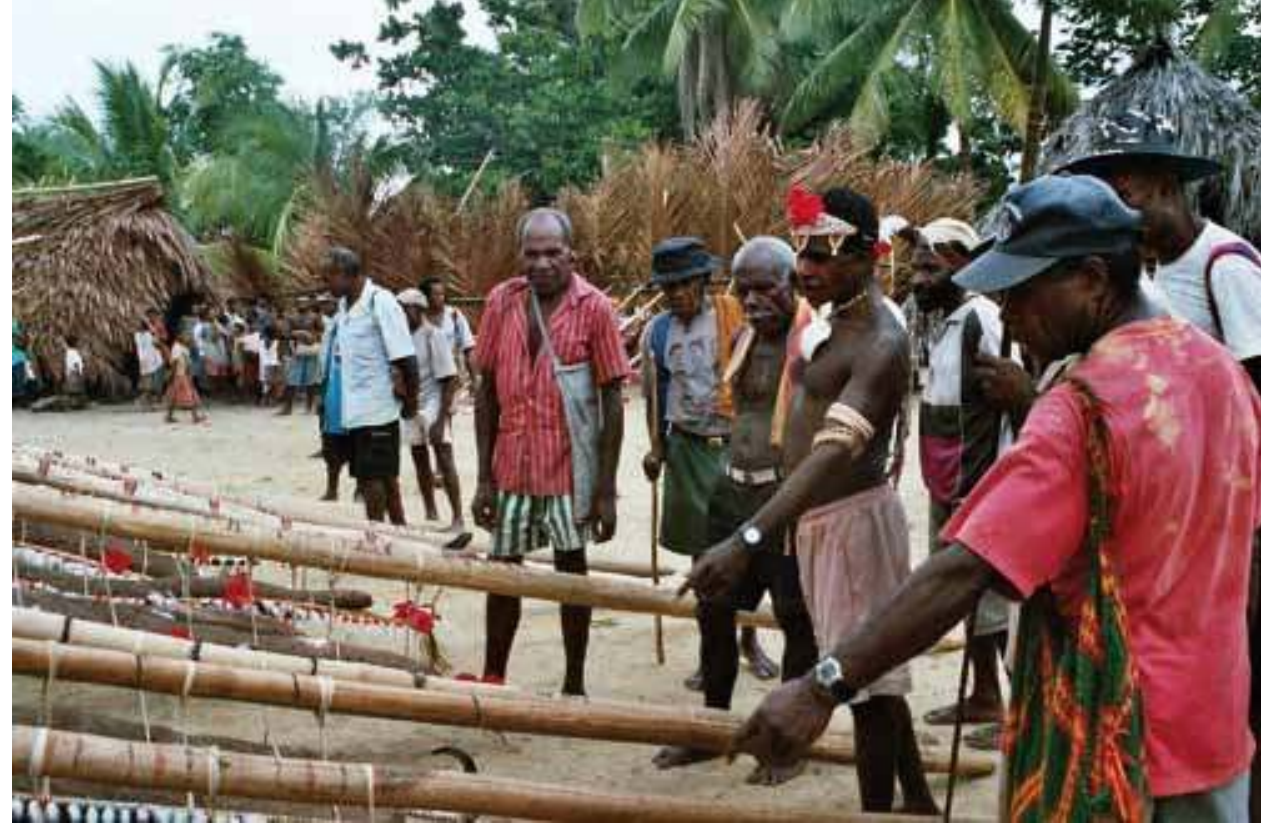

- L. Coupaye nie, qui dure une journée entière et la nuit suivante, inclut deux moments essentiels: la sortie et l'évaluation des waapi, puis la danse de nuit. Une fois la cérémonie finie, les waapi intègrent les différents circuits d'échanges coutumiers, principalement les compensations matrimoniales et funéraires, ainsi que le règlement des disputes. Autrefois, les waapi étaient également au centre des échanges entre partenaires des deux moitiés cérémonielles (indépendantes des clans; cf. Kaberry 1941 ; Forge 1990), soit au sein du même village, soit appartenant à deux villages rivaux. Cette pratique semble avoir cessé en raison des conflits parfois sanglants que ces échanges agonistiques pouvaient générer, aux dires des habitants eux-mêmes, particulièrement pour les échanges impliquant deux villages (Forge 1990 : 161-163). Ce rôle d'items produits exclusivement par les hommes pour des échanges cérémoniels de nature agonistique a très tôt été à la source des premières analyses des waapi comme symboles au centre d'un culte phallique (Kaberry 1941 : 355-356; Forge 1970 : 271 , 1990 : 162-163).

J'ai décrit ailleurs plus en détail la chaîne opératoire et les éléments principaux du système technique considérés comme requis pour l'obtention d'un tubercule digne d'être présenté lors d'un Waapi Saaki (Coupaye 2009, 2010, 2012). Au premier plan de ces étapes nécessaires se trouvent les techniques de Yakët, qui recouvrent l'ensemble des prescriptions et proscriptions alimentaires et comportementales qui transforment et préparent le cultivateur au contact des tubercules, mais aussi au commerce nécessaire avec des collectifs d'entités non-humaines résidant sur le territoire, ainsi qu'avec les autres membres humains de la communauté. Conformément à la formule de Mauss (1950 [1936]), l'ensemble de ces gestes techniques et de ces rites s'assemblent en une série d'actes « traditionnels » et « efficaces » - selon les critères des acteurs eux-mêmes - de transformation des matières, des corps et des substances mobilisés lors des différentes étapes du processus. L'usage de cette formule combinée à celui de la chaîne opératoire permet de réaliser une sorte de transect dans la réalité vécue des habitants de Nyamikum, montrant les rapports multiples entre personnes, choses, entités et techniques, et ce sur l'ensemble du cycle horticole, culminant avec la cérémonie annuelle, Waapi Saaki, et se poursuivant par des échanges jusqu'à la consommation et la replantation.

Mais si les waapi sont intentionnellement données à voir, c'est notamment parce qu'elles sont les parties visibles, concrétisées, de ces réseaux de relations manipulés par 


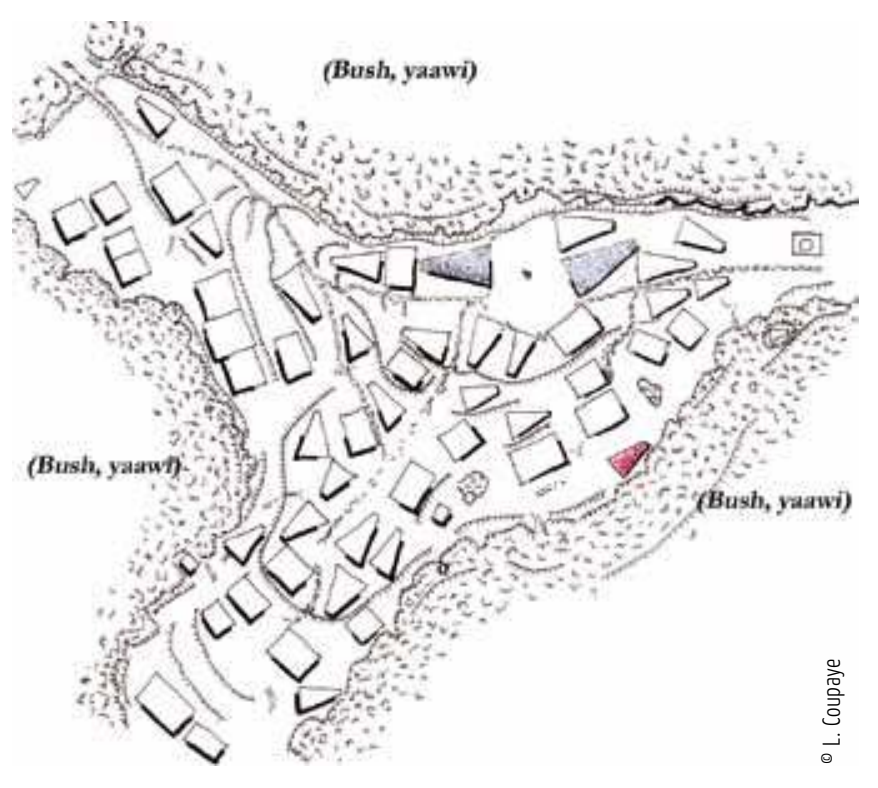

leurs cultivateurs et du talent dont ces derniers ont fait montre pour obtenir un résultat non seulement adéquat, mais aussi spectaculaire (Coupaye 2013). Et parmi les nombreux éléments tangibles et fondamentaux se trouvent des objets, qui sont eux invisibles, cachés et secrets. Je veux parler ici des pierres sacrées dont l'existence est tenue secrète - ce qui explique leur relative absence de la littérature ethnographique, notamment chez Kaberry et Forge, quoique plus présentes dans les travaux de Gerrits $^{7}(2012$ [1977]) pour le village de Bongiora, ceux de Huber-Greub (1988) pour le village de Kimbangwa, d'Hauser-Schäublin (1989a) pour le village de Kalabu et chez Aufenanger (1972). Cette position particulière des pierres au sein du système technique à l'origine des waapi s'explique peut-être mieux à la lumière l'anecdote suivante.

Relevé du hameau de Kumim (village de Nyamikum, mars 2002)

Ce diagramme présente le relevé du hameau, en son état en mars 2002. Le relevé a été exécute avant qu'on puisse en voir les détails sur Googlemap, aux coordonnées suivantes: 3.623355,143.078301 Le hameau est localisé comme souvent, sur le sommet d'une crête, bordée par la brousse (yaawi, terme aussi utilisé pour les jardins) Les deux bâtiments colores au nord correspondent aux maisons de réunion, localise à l'est et à l'ouest de l'aamë, la place cérémonielle. Au centre de cette dernière se trouve l'amas de pierre mbaapmu-taakwa (cf. fig. 12-16). Les bâtiments de plan trapézoïdal correspondent pour la plupart aux greniers à ignames (dont l'un contient la pierre sacrée). Les bâtiments de plan quadrangulaire correspondent aux maisons d'habitation sur poteaux.

(Fig.4)

\section{Des pierres pour leurs jardins}

En juillet 2003, j'assistais au Waapi Saaki de Balokwil, l'un des hameaux principaux de Nyamikum. Lors d'une pause dans la série de discours suivant la sortie des waapi, l'un des hommes éminents du hameau $\left(N e \ddot{m} a^{n} d u^{3}\right)$ m'invita à le suivre hors de l'aamë (place cérémonielle) et m'amena devant l'un des nombreux greniers à ignames composant le hameau. D'une taille modeste, tout au plus six à sept mètres de long pour un peu plus de deux de haut, le bâtiment présentait une structure que l'on retrouve depuis les maisons de jardins jusqu'aux fameuses kura ${ }^{m} b u$ (les maisons cérémonielles, ou Haus Tambaran en Tok Pisin, analysées par Gerrits 2012 [1978], ainsi que par Smidt et McGuigan, 1994), en passant par les « chambres coucher » (avant qu'elles ne soient remplacées par les maisons «modernes » sur poteaux): un toit à double pente tombant jusqu'au sol, en pente douce vers l'arrière, sur plan trapézoïdal et, ici, fermé à l'avant et à l'arrière. Rien ne permettait de discerner le grenier en question de la douzaine d'autres composant ce hameau - ou ceux des autres (Figures 4 et 5). Posant sur moi un regard oblique, mais intense, le Nëma ${ }^{\text {nd }}$ u me déclara avec gravité: « Tu es venu ici pour comprendre le mayëra des grandes ignames... » Pointant le grenier du menton, il asséna: « Ceci est le mayëra des grandes ignames. ».

Ce moment de révélation dramatique marquait un contraste frappant avec ce qui se passait sur l'aamë. D'abord, le terme utilisé par mon guide, mayëra, renvoie à un univers complexe de choses sacrées et secrètes qui ne peuvent être exposées publiquement. Mayëra peut tout aussi bien s'appliquer à des images, des savoirs ésotériques, des savoir-faire, ou bien sûr des pratiques et des artefacts, dont l'existence est connue de toutes et de tous, mais dont le contenu n'est pas explicite. Ici, le mayëra faisait référence à un artefact caché à l'intérieur même du grenier, une pierre sacrée, ainsi qu'à l'ensemble des pratiques qui entourent cette dernière.

C'était loin d'être la première mention faite à cette pierre. De fait, mes amis de Nyamikum mavaient décrit en détail son rôle lors de la première partie de mon terrain 
en 2001-2002. Mais la révélation faite par le Nëma ${ }^{n} d u$ à cette occasion confirmait avec force son caractère central et, notamment, sa nature de «prototype »invisible et pourtant présent - des ignames exposées à quelques dizaines de mètres de là, aux yeux de tous. Les waapi, n'apparaissent plus seulement comme des images données à voir d'une capacité reproductive, mais aussi de pratiques coutumières fondamentales - voire fondatrices. Comme on va le voir les waapi constituent - particulièrement dans la formulation de mon guide - les contreparties visibles de la pierre.

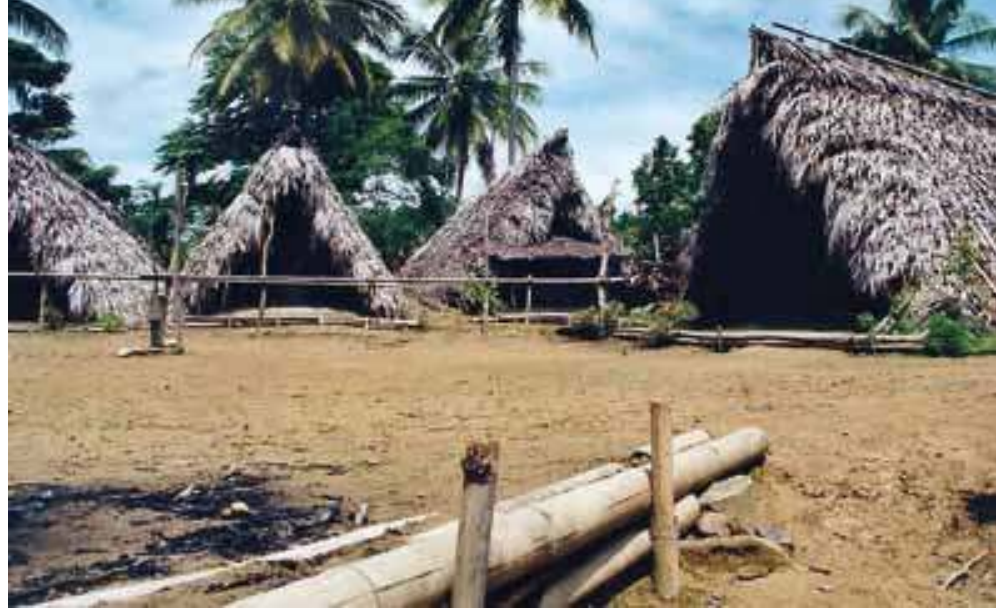

๑ L. Coupaye

Selon les descriptions ${ }^{4}$ faites par mes amis de Nyamikum, les pierres peuvent varier de forme, de matériau et de dimension, mais il semble qu'il s'agisse toujours d'un minéral, le plus souvent de forme allongée, pouvant atteindre jusqu'à un mètre ${ }^{5}$. Il existe une pierre par grande catégorie de nourriture, regroupant souvent plusieurs types de plantes, distinguant parfois entre eux des cultivars de la même espèce. Ainsi m'a-t-on fait mention pour Nyamikum ${ }^{6}$ d'une pierre pour les Maambutap, le cultivar de grande igname le plus prestigieux; une pour la $W u^{n} j \ddot{e}^{m} b u$, le cultivar de $D$. alata venant tout de suite après elle; une pour les petites ignames; une pour les taros, les bananiers, le palmier-sagoutier et le sucre de canne; une pour les arbres à pain et les cocotiers. Il semble qu'autrefois étaient en usage des pierres dédiées à d'autres catégories, différentes des plantes, notamment une pour les oiseaux, une pour les cochons. Nous reviendrons sur ces catégories, mais pour l'instant notons que chaque village semble donc être en possession d'une collection de pierres destinées à jouer un rôle essentiel dans les pratiques associées à la culture de diverses espèces vivantes majoritairement destinées à être consommées.

Chacune de ces pierres est, aujourd'hui encore, sous la tutelle d'un clan particulier, clairement identifié et connu de tous comme tel. Cette répartition des pierres entre les clans indique une forme, sinon de spécialisation, du moins de répartition des tâches entre les différents clans d'un même village. Le clan associé à une pierre donnée a pour responsabilité de la conserver - y compris durant les migrations - ainsi que de lui assigner un « gardien », dont la charge est non seulement d'entretenir la pierre et l'espace qui lui est consacré, mais également d'exécuter les rituels et les pratiques qui lui sont associés. Appelé $K a^{n} j a t u^{n} d u$ (lit. « Homme-joint-de-barrière »), ce gardien, doit se soumettre à un Yakët ${ }^{8}$ extrêmement strict dont le rôle principal, outre d'augmenter l'efficacité des rites à accomplir, est de protéger son corps de la proximité potentiellement dangereuse des effets de la pierre. La position de gardien est occupée durant plusieurs années consécutives par la même personne, dont l'identité est tenue secrète, jusqu'au moment où celle-ci est remplacée, soit pour lui permettre de « se reposer » soit parce que son efficacité n'a pas été jugée suffisante. Il est explicitement comparé au conducteur d'un bus qui doit mener à bien son voyage. La pierre en question est conservée dans le hameau-résidence du clan, et il semble donc qu'elle soit installée dans le grenier à igname appartenant au gardien désigné.

C'est à l'arrière de ce grenier qu'est installé une sorte d'autel, dans un espace séparé du reste et caché à la vue par une barrière. La pierre dédiée aux ignames du type Maa $a^{m} b u t a p$ (Figure 6) est dite reposer sur une infrabase de feuille de palmier sagoutier, posée à même le sol et entourée, en particulier, d'anneaux de coquillage - on y reviendra. Le reste se 


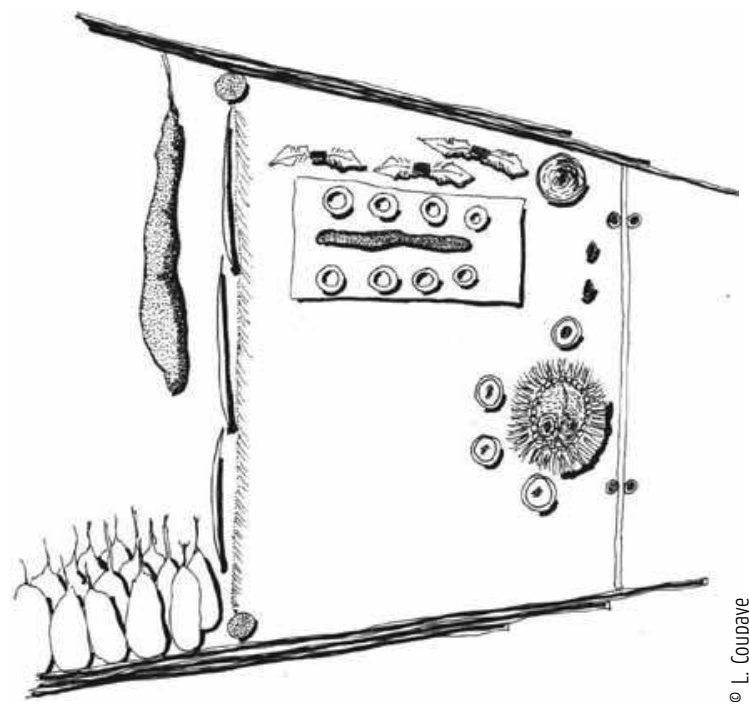

Plan de l'intérieur d'un grenier où est installé l'autel.

\section{Masque mbaapa durant une cérémonie célébrant le début du} revêtement de la route Maprik-Wewak (09.04.02)

Autrefois ce type de masque intervenait dans la plupart des cérémonies d'initiation, jouant un rôle de gardien et de contrôle de la foule. Ici, durant cette cérémonie plus « moderne », le masque brandissant un javelot, est chargé de faire dégager la route pour les autres danseurs, en menaçant les importuns de son arme. Chaque clan était censé posséder un de ces masques, et chacun d'entre un portait un nom particulier. Le terme mbaapa signifie parfois « tête », ou « chef » (comme dans le terme : « couvre-chef »). Ces masques font partie des éléments composant les autels à pierre pour les Mazmbutap (cf. fig. 6). (Fig. 7)

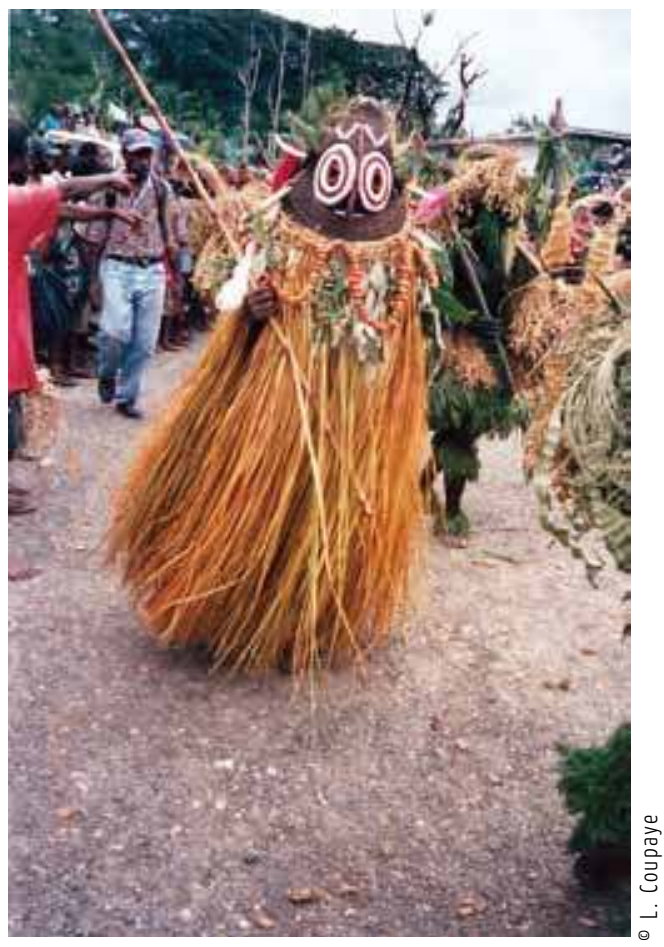

compose semble-t-il d'une série d'éléments qui incluent, notamment le masque ${ }^{m}$ baapa $^{9}$ (Figure 7) appartenant au clan, suspendu et entouré de bols en noix de coco.

Seul le gardien de la pierre, en particulier durant la pousse des ignames, est autorisé à s'en approcher et à la toucher lors des rites - ce qui pourrait le blesser physiquement s'il ne s'était pas rigoureusement conformé aux règles imposées par son Yakët. Les rites consistent principalement à, d'une part, laver de la pierre à l'aide d'un liquide composé d'eau et de la sève de plantes particulières et, d'autre part, à placer autour d'elle les anneaux de coquillage. Il lui revient également de remplacer les éléments de l'autel lorsque ces derniers sont défraîchis (notamment la base de feuilles sur laquelle repose la pierre). Ces deux opérations ont lieu, dit-on, au début d’un nouveau cycle horticole, affectant les plantes et les jardins concernés.

\section{Des pierres-reliques}

L'origine des pierres dédiées aux ignames Maa $a^{m} b u t a p$ et $W u^{n} j^{m} e^{m} b u$ sont chacune associées à un héros culturel: Wapikany pour la première, Sërapwany pour la seconde. Encore une fois, l'espace manque ici pour rendre compte de l'ensemble de ces mythes - dont on trouve par ailleurs de nombreuses variations selon les villages ${ }^{10}$ - mais dans les deux cas, les pierres apparaîssent comme les reliques des deux personnages. Ainsi la pierre associée à la $W u^{n} j^{m} e^{m} b u$ est l'ornement de nez de Sërapwany qui, transformé en cochon destructeur de jardins, finit par être chassé et tué par son père. Quant à la pierre dédiée à la Maa $a^{m}$ butap, son origine est encore plus parlante.

Wapikany est en effet « l'inventeur » de la culture des grandes waapi et notamment des interdits sexuels composant le Yakët. Ayant surpris son père en pleine transgression, Wapikany prit la fuite, aussitôt pourchassé par celui-ci. Mais lorsque son père parvint à le rattraper et à poser sa main sur son bras, celui-ci se détacha du corps de Wapikany, qui poursuivit sa course. Son père laissa tomber le membre pour continuer à le poursuivre. La scène se répéta, Wapikany semant ainsi des morceaux de son propre corps sur l'ensemble du territoire qui s'étend d'Apangai dans l'ouest et à Kalabu dans l'est ${ }^{11}$ (Figure 8). Une fois détaché du corps de Wapikany, chaque tronçon se transforma en une pierre sacrée.

Il y a bien sûr beaucoup à dire sur ces deux mythes ${ }^{12}$ et je ne mentionnerai ici que quelques points importants pour ma démonstration. D'abord, l'analogie des morceaux du corps de Wapikany avec des tronçons d'ignames tels que ceux utilisés pour la plantation: la dispersion des fragments de Wapikany sur le territoire évoque la dispersion des boutures qui, une fois replantées, donnent des plantes qui ne sont ni plus ni moins que de nouvelles instanciations du même individu végétal 
(Burkill 1951 : 444). Puis, bien sûr, l'idée du lien entre la culture des Maambutap et le Yakët associé, notamment les interdits sexuels. Ensuite, le fait que la course de Wapikany suit peu ou prou la direction des migrations des clans, telle qu'elle est narrée par les Nëman $d u$. Enfin, le nom même du héros « Wapikany », « grande igname-roseau ${ }^{13}$ relie directement le personnage aux waapi. Tous ces éléments indiquent l'importance cosmologique de la pierre, cosmologie dont les sources orales sont connues pour être rares chez les Abelam (Forge 1965 : 24), mais dont l'existence finit par se manifester de manière visuelle et matérielle, c'est-à-dire majoritairement non-verbale ${ }^{14}$. De fait, ici, si le mythe fournit des indices assez clairs, les commentaires des narrateurs ne s'étendent pas à une exégèse élaborée (en tous cas pour ceux que j'ai pu rencontrer), préférant, selon l'usage, laisser l'auditoire tirer ses propres conclusions.

On peut conclure ici sur la nature de reliques de ces objets, indices de la permanence de la présence fertile du héros culturel, présence minéralisée, donc immuable, et dispersée/répartie dans les différents villages.

\section{Des pierres-centrales... et centrales}

L'espace manque ici pour une description et une analyse plus complète des autels à pierre. Toutefois, les informations réunies au sujet de ceux dédiés aux Maambutap, permettent de penser que le rôle qui leur est assigné dans la culture des ignames est de transmettre aux groupes et aux individus auxquels ils sont « connectés » une forme, sinon de fertilité, du moins d'énergie capable d'influer sur la pousse de la plante ${ }^{15}$. Ce rôle est rendu possible par la présence sur l'autel de la pierre d'objets qui relient, ou « connectent», cette dernière aux jardins. Ces objets sont, d'une part, les bols en noix de coco disposés autour du masque et, d'autre part, les anneaux de coquillage posés autour de la pierre elle-même. On va le voir, cette installation semble reproduire à une échelle réduite les relations entre les clans ainsi que les accords passés avec des membres d'autres villages - interprétation confirmée par mes interlocuteurs.

Les bols en noix de coco sont censés représenter (au sens de « rendre présents ») les clans composant le village - le cocotier étant, comme l'a démontré Huber-Greub (1988), un marqueur d'autochtonie. Leur présence autour du masque mbaapa du clan gardien de la pierre semble positionner ce dernier au cour de la circulation de l'énergie qui permet la reproduction des grandes ignames Maambutap. Quant aux anneaux de coquillage, ils sont donnés par des représentants d'autres villages. Placés au plus près de la pierre, les gens indiquent qu'ils assurent la transmission du pouvoir de cette dernière aux jardins situés au-delà des limites de Nyamikum.

Les anneaux de coquillage occupent une place centrale dans les rapports entre les groupes. Nommés yëwaa (terme aussi utilisé pour désigner la monnaie moderne), ces anneaux, comme les Maa ${ }^{m}$ butap, ne circulent que lors des compensations matrimoniales et funéraires ou pour 


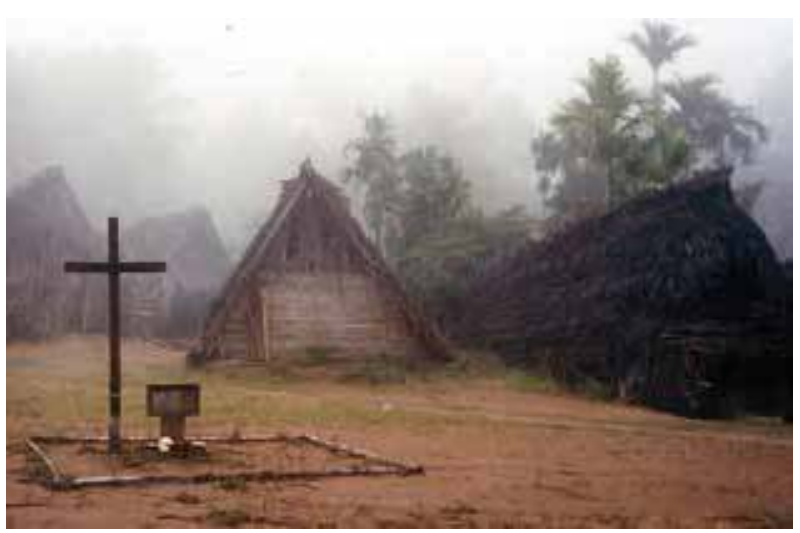

(Fig. 12)

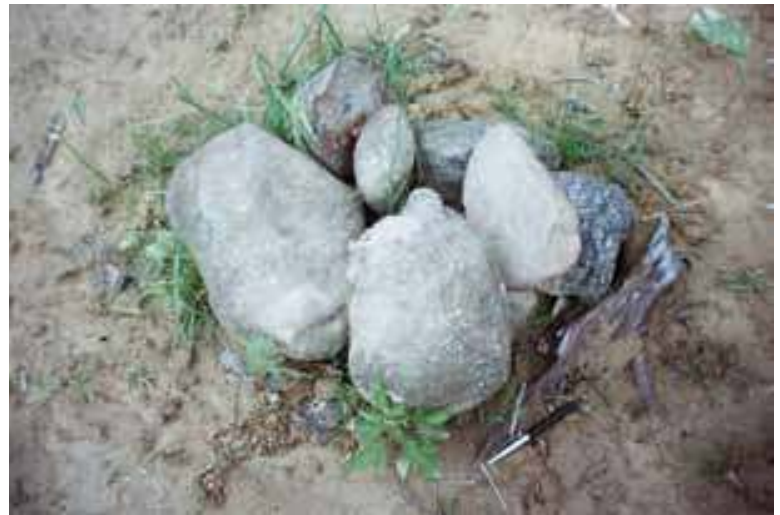

(Fig. 13)

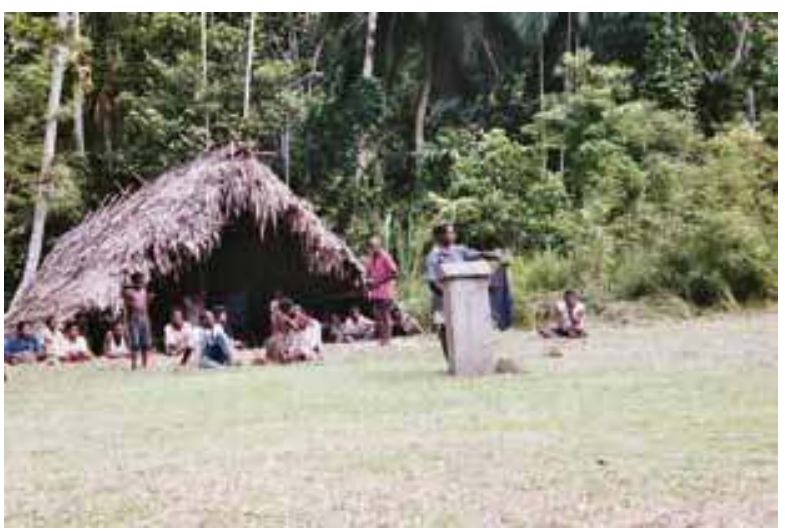

(Fig. 14)

(Fig. 16)

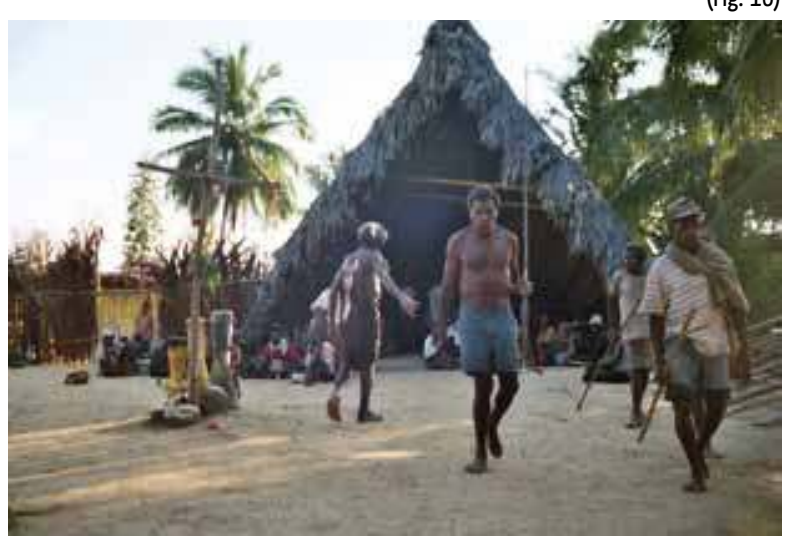

\section{« Araignées-Lunes » et Places cérémonielles}

Photo de mbaapmu-taakwa placées au centre des places cérémonielles (aamë) de plusieurs hameaux de Nyamikum. Ces amas de pierres sont généralement composés de pierres trouvées dans les rivières (fig. 13) à la suite d'un rêve, et marquent le cœur du hameau. Certains sont accompagnés de croix, indiquant leur association avec le catholicisme (fig. 12, 15 et 16). D'autres sont accompagnés d'une stèle en ciment (figures 12 et 14) portant le nom d'un membre décédé éminent du hameau. Les mbaapmu-taakwa sont au cœur des discours publics, soit durant les réunions de village (fig. 14) soit durant les occasions cérémonielles (fig. 15-16). (Fig. 12-16)

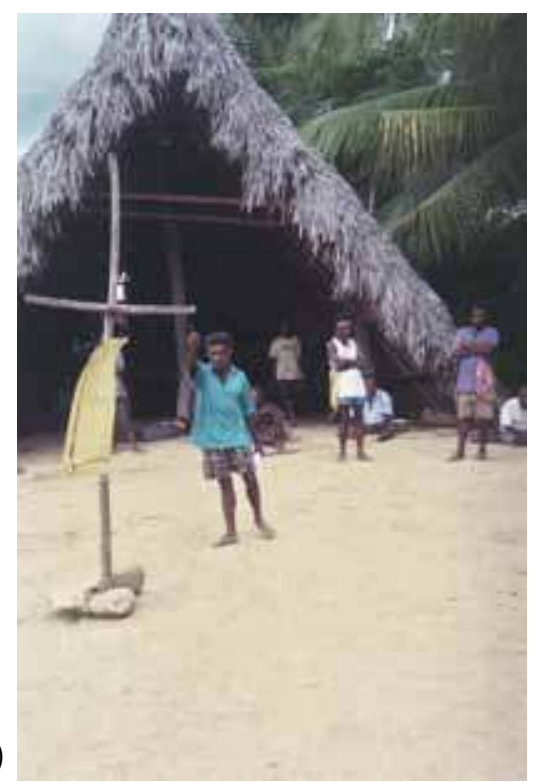




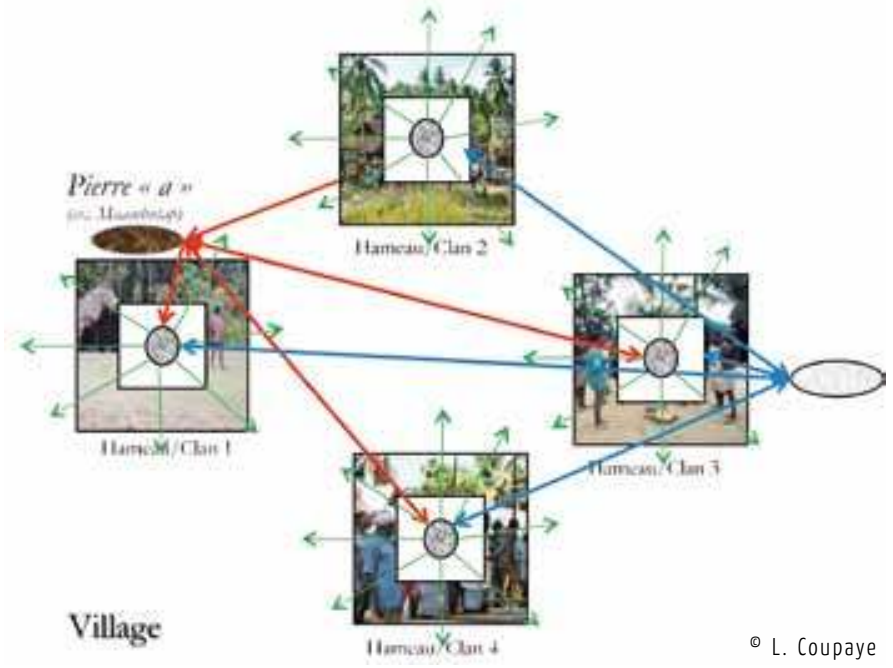

Le « réseau d'énergie »

Le niveau du village : Ce diagramme représente quatre hameaux 1, 2, 3 et 4, d'un même village, chacun pourvu d'un aamë (place cérémonielle) au centre duquel se trouve une mbaapmu-taakwa (cf. Fig. 12-16). Le " hameau 1 » est celui dans lequel se trouve conservée la pierre « a » (par exemple dédiée aux Maambutap). Le « hameau 3 » conserve la pierre « $b$ » (ici, dédiée au Wunjembu). Chacune de ces deux pierres est « connectée » à la mbaapmu-taakwa de tous les hameaux du village. Les discours alimentent la pierre, et celle-ci « redistribue » son pouvoir, via mbaapmu-taakwa à tous les hameaux du village (en rouge, le pouvoir pour les Maambutap; en bleu le pouvoir pour les Wunjembu). Le pouvoir de la pierre est à son tour transmis aux différents jardins (flèches vertes). (Fig. 17)

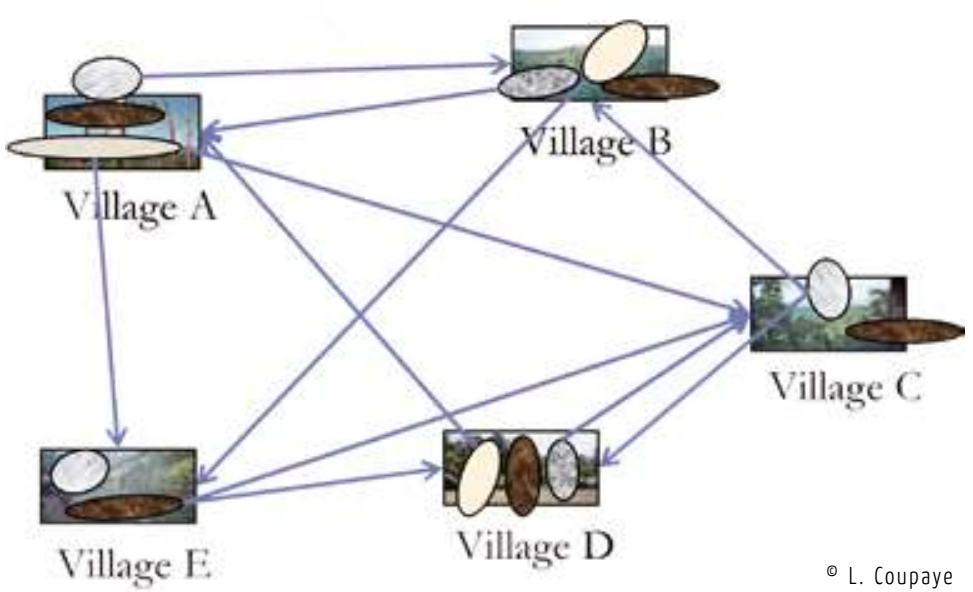

Le « réseau » régional

Ce diagramme représente de manière schématique les réseaux de relations de « soutien » que les villages peuvent développer entre eux, à partir des pierres qu'ils possèdent. Chaque village (rectangles) possède un jeu de pierres (représentées par les formes oblongues), chacune « dédiée » á une catégorie spécifique de nourriture. Un village peut alors demander le « soutien » d'une de ses pierres pour une culture particulière à un ou plusieurs villages voisins, selon leur réputation et leurs succès lors des récoltes précédentes. Ainsi, par exemple, le village « $E$ » soutien les villages « $A$ », « $C$ » et « $D$ » pour la culture des Maambutap (pierre brune).

L'autel de la pierre du village « $E$ » contient alors des anneaux donnés par ces villages. En revanche, ce même village « $E$ » reçoit le soutien du village « $A$ » pour - disons - la culture de ses Wunjembu (pierre oblongue grise) et du village « B » pour les arbres-à-pain (pierre beige). (Fig. 18) 


\section{Une toile de connexions}

J'ai demandé mes interlocuteurs si le fait que le terme d'« araignée-femme » donnait à penser que l'ensemble des pierres formait une toile (wut ${ }^{18}$ en Ambulas) était fortuit. Leur réponse positive, « ça n'est pas une toile, mais ça fonctionne pareil », suggère des similarités, sinon d'essence, du moins de propriétés (Figures 17 et 18).

L'existence de la pierre et le secret qui l'entoure éclairent le phénomène des grandes ignames décorées sous un jour autre que celui de simples symboles phalliques employés lors d'échanges agonistiques. Tout d'abord, la nature de relique de la pierre confirme son caractère sacré occupant la position de ce qu'Annette Weiner (1992) et Maurice Godelier (1996) ont analysé comme étant des « possessions inaliénables » : transmise de génération en génération, elle ancre l'identité du groupe qui la possède dans un objet d'une permanence matérielle tangible. Par ailleurs, la possession de ces reliques implique également des rapports politiques entre les clans et entre les villages, représentant l'irreprésentable et permettant ainsi la coalescence de noyaux d'imaginaire dont parle Godelier (1996 : 151-152 ; 239-249).

La répartition des pierres entre les villages dessine une situation dans laquelle chacun est en possession d'une série particulière, tel un joueur disposant d'une « main » que l'histoire des ancêtres et des migrations lui aurait fourni. Ceci implique que tous les villages n'ont pas toutes les pierres, mais un certain jeu. Cette répartition oblige ainsi un village $\mathrm{A}$ à entamer des négociations afin d'obtenir le soutien d'un village $\mathrm{B}$, connu pour posséder la pierre correspondante aux espèces pour lesquels A désire obtenir des résultats particuliers. Et quand bien même la majorité des villages auraient en leur possession au moins une pierre à Maa $a^{m} b u t a p$, les Waapi Saaki sont l'occasion de voir quel(s) village(s) sont capables d'obtenir les waapi les plus impressionnantes. D'où l'importance d'assister aux cérémonies des autres villages et des conciliabules ayant lieu durant la nuit de la cérémonie, afin de pouvoir donner un anneau pour que celui-ci soit installé sur l'autel correspondant, et d'avoir accès à ce mayëra si efficace.

Par ailleurs, la notion de mayëra permet de valider, de protéger et de contrôler le pouvoir qu'un individu ou qu'un clan pourrait tirer de la pierre dont il a la charge. Mayëra réfère, en effet, à ce domaine complexe de pratiques et de savoirs tirant son origine du pouvoir des ancêtres validé par les réalisations passées du clan et du village lui-même. Le caractère sacré de la pierre fixe l'identité du clan et sa permanence par son caractère de relique transmise de génération en génération. Cette origine mythique est la source d'un pouvoir dont bénéficie plus d'un individu ou d'un clan, mais un ensemble de clans défini comme formant un village. Comme indiqué à l'instant, en se référant aux travaux de Weiner (1992) et Godelier (1996) sur les objets inaliénables, on peut inférer que les jeux de pierres possédés par un village sont donc les ancrages mythiques de l'unité politique du village et de sa reproduction.

Le mayëra de la pierre protège également contre les effets des intrigues politiques dans lesquelles baigne l'ensemble des villages Abelam. L'identité réelle du Kanjatun $d u$ en charge de la pierre pour la période donnée est tenue secrète par un jeu de leurres et de rotations entre un nombre indéfini de candidats potentiels. Le $K a^{n} j a t u^{n} d u$ est toujours choisi parmi un groupe d'individus reconnus comme possédant les qualités requises à l'entretien de la pierre. Du coup, les tentatives d'assassinat (par des moyens physiques ou mystiques, par le recours à la sorcellerie) par les membres d'autres villages, jaloux du pouvoir et de l'influence qu'une communauté particulière pourrait posséder grâce à ses succès en matière de culture des ignames, n'ont qu'une cible mouvante. 
On retrouve ici l'un des principes majeurs de la socialité Abelam. En effet, nul individu ne peut se vanter de ses privilèges rituels, de son rôle politique central ou de ses talents qu'il s'agisse de talents de cultivateur d'ignames ou de maître en matière de rituels - sous peine de devenir la cible privilégiée d'attaques de rivaux internes ou externes au village. Cette règle s'applique aussi à ceux qui ont en charge une pierre (avec moins de risques d'être attaqué par les membres de sa propre communauté, bien que rien ne prouve que cela ne se soit pas produit par le passé).

Ceci permet également d'éviter que les clans chargés des pierres à Maambutap, occupent une position privilégiée au sein de leurs villages respectifs. En effet, il apparaît que les pierres semblent être, au final, considérées moins comme étant la propriété exclusive de leurs clans gardiens, que comme des reliques dont l'intendance leur aurait été confiée pour le bénéfice du village en entier. Les discours des Waapi-Saaki insistent notamment bien davantage sur le rôle central que le village en entier peut jouer dans la géopolitique Abelam de par sa capacité à obtenir les plus belles Maa butap, plutôt que sur l'importance du clan particulier en charge de la pierre. Cette centralité se manifeste notamment par la quantité de requêtes adressées au village pour qu'il apporte son soutien à des cultivateurs d'autres localités venus en faire la demande, et qui fourniront un anneau de coquillage à placer auprès de la pierre. Il semblerait alors que, ce qu'un village met en avant, c'est la richesse de sa socialité, non pas uniquement à son seul bénéfice, mais une richesse qui rayonne au-delà de ses frontières et participe à la reproduction (par la nourriture, entre autres) de la région en entier.

Pour terminer, le rapport pierre-igname met en lumière un contraste matériel et idéel entre une source imputrescible et unique et ses images sous forme d'ignames dont la permanence est assurée par leur réplication en de nombreux exemplaires à chaque nouvelle saison. Les waapi apparaissent alors comme la manifestation, voire l'émanation principale, non pas uniquement du pouvoir de la pierre, mais aussi de pratiques de mobilisation de collectifs complexes et dangereux dans des rapports toujours sociaux qui impliquent, outre des humains, des entités et des substances, puissantes parce qu'atemporelles, durables et par-dessus tout, intimement liées aux groupes qui sont en permanentes négociations avec elles.

Ce sont ces rapports-là, peut-être plus encore que la pierre elle-même, qui semblent émerger au cœur du phénomène. Il peut en effet advenir que son pouvoir faiblisse jusqu'à disparaître, comme cela semble avoir été le cas pour les pierres à cochons et celles aux oiseaux. Réciproquement, un clan ayant perdu sa pierre - que celle-ci ait été détruite par l'ennemi, ou désacralisée - continue bien d'exister, mais ne semble pas pouvoir la remplacer par une autre ${ }^{19}$.

\section{Irremplaçables objets, irremplaçables pratiques}

Les pierres occupent ici plusieurs rôles dans lesquels elles sont irremplaçables. Pour les Abelam, il s'agit de reliques d'ancêtres qui non seulement valident la légitimité territoriale et politique des clans, mais autour desquelles se focalisent aussi des pratiques qui lient la culture des jardins, la socialité et les relations politiques entre les villages. Ces pratiques sociotechniques sont jugées essentielles aux activités centrales de la communauté 
c'est-à-dire la culture des jardins et les échanges coutumiers. Et ceci à tel point que, contrairement aux initiations, les entités non-humaines qui leur sont associées ne semblent pas critiquées par les différentes confessions chrétiennes présentes à Nyamikum ${ }^{20}$. Les pierres, et notamment celles dédiées au Maambutap, sont les nœuds autour desquels partent les connexions multiples qui tissent l'ensemble du territoire Abelam, et la source de ce qui permet de créer de la nourriture et de la socialité. Elles sont enfin les contreparties, invisibles, certes, mais permanentes et inaliénables, des grandes ignames exposées, qui elles, sont faites pour être échangées.

Pour nous, ces pierres invitent à penser conjointement les pratiques sociotechniques, les rites, mais aussi les notions de visibilité, de matérialité, de reliques, d'images, de fertilité et de rapports politiques. Jusqu'à leur mention, on pouvait en effet interpréter les ignames sous l'angle de ce qu'elles donnent à voir immédiatement, et des usages qui en sont faits - de richesses à échanger, y compris d'un culte phallique ${ }^{21}$ qui opposerait les groupes entre eux. Lajout des pierres dans l'analyse illustre que pour comprendre la matérialité des ignames, il faut la mettre en relation avec d'autres objets et pratiques, sur lesquels les gens peuvent être peu explicites, mais dont l'absence est parfois plus parlante que leur exposition. La nature de secret entourant la pierre et ses rituels devient alors l'une des composantes essentielles d'un véritable jeu de stratégie et d'alliance, liant les clans et les villages entre eux dans un réseau dans lequel des objets permanents autour desquels se tissent, au moyen d'items mobiles (les ignames?), des relations entre les groupes.

Si besoin était, on verrait combien les pierres et les ignames forment bien plus qu'un phénomène permettant d'illustrer ce qu'est un réseau d'acteurs. Mais une toile de relations dans laquelle on peut penser, à partir de la formule d'Haudricourt (1987 [1962]), la manière dont les groupes associent traitement des plantes, traitement de la culture matérielle et traitement d'autrui.

\section{NOTES}

Photo d'ouverture: Anneaux marquant une alliance

1. La même pratique se retrouve dans un certain nombre de groupes voisins, bien que linguistiquement différents ( $c f$. entre autres Tuzin 1972, 2002).

2. Nyamikum est situé juste au nord-est de Maprik et comptait, à l'époque de mon terrain entre 2001 et 2003, quelques 2000 habitants.

3. Littéralement « grand homme », ce terme est utilisé pour désigner à la fois les frères aînés et les hommes ayant acquis un statut notoire par leurs talents d'orateur, de cultivateurs de waapi et autrefois de guerriers et d'experts en matière de rituels. J'utilise des capitales pour les noms de positions (Nëman $d u$ ou Kanjatunndu, cf. plus bas) ainsi que d'institutions (telles que le Yakët).

4. Pour des raisons de discrétion, je ne peux mentionner les sources exactes de mes informations. Comme on le verra plus bas, le secret entourant les pierres concerne en définitive moins leur existence, ou même les pratiques, que le lieu exact dans lequel elles se trouvent ainsi quel'identité des personnes qui en ont la charge. Cependant ce secret moblige à ne faire référence ici qu'à des descriptions faites par les membres de la communauté de Nyamikum étant,ou ayant été eux-mêmes directement impliqués dans l'entretien et les rituels associés à la pierre. Par chance, il s'agissait de la pierre responsable des Maam butap, les principaux cultivars de grandesignames.

5. Dans d'autres cas, il peut s'agir d'un coquillage d'une taille exceptionnelle. Aufenanger (1975 : 169) mentionne également l'utilisation d'une lame de hache.

6. Les données suivantes ne sont valables que pour Nyamikum, village dans lequel j'ai pu accéder à ces informations. Les descriptions faites par Aufenanger 
(1972 : 169; 1975 : 144-147), Huber-Greub (1988: 147-148; 182-187), Hauser-Schäublin (1989a: 186189) et McGuigan (1992 : 212-214) diffèrent sensiblement de celles auxquelles j'ai eu accès, différences qui peuvent également être dues aux variations parfois radicales existant entre les villages Abelam. Les noms vernaculaires des objets comme des rôles que je vais décrire peuvent varier considérablement d'un village à l'autre. Pour des raisons d'espace évidentes, la comparaison adéquate de ces différentes données ne peut se faire ici.

7. Au moment de la rédaction de cet article, l'ouvrage de Gerrits n'avait pas encore été publié et je n'avais pu y avoir que brièvement accès que quelque mois avant mon séjour en 2001. Aussi, le présent article n'a pu bénéficier de l'excellente documentation fournie par Fred Gerrits.

8. Rappelons que ce terme recouvre les prescriptions et proscriptions alimentaires et comportementales auxquelles doivent se soumettre les cultivateurs d'ignames.

9. Plus communément transcrit comme «baba ». Cf. Hauser-Schäublin 1984, et les exemplaires exposés au Musée du Quai Branly (Inv. 72.1962.3.3).

10. Pour Wapikany: Huber-Greub 1988 : 147-148; $286-$ 287; McGuigan 1992 : 309-312. Pour Sërapwan, cf. : Huber-Greub 1988 : loc.cit., Hauser-Schäublin 1989a: 198-199. Aufenanger (1975 : 107) mentionne également une origine liée au soleil.

11. La course de Wapikany s'achève dans différents endroits, selon les versions. À Nyamikum, Wapikany finit par se jeter dans le lac Imbiap.

12. Voir notamment l'analyse faite par McGuigan 1992, loc. cit.

13. Huber-Greub (1988 : 147) donne une version alternative du nom du personnage, Wapilen - que j'ai aussi retrouvé à Nyamikum sous la forme Wapilany. À noter que lany signifie sperme.

14. Voir l'analyse remarquable d'Hauser-Schäublin (1989a: 298-299) sur l'organisation spatiale de la place cérémonielle aamë et du système d'orientation qu'elle matérialise, impliquant un axis mundi vertical recoupant un axe horizontal reprenant la course du soleil.
15. Je n'ai malheureusement pas d'information sur les autres pierres, ce qui ne permet pas d'affirmer avec certitude que l'énergie en question agit sur les espèces elles-mêmes (plantes, animaux) ou sur les humains qui les cultivent/chassent. S'agit-il donc d'une énergie qui agirait sur la fertilité des plantes - ou leur fécondité - ou bien sur l'efficacité des processus dans lesquels les humains se lancent? Comme on va le voir, il est possible que ce soit cette dernière possibilité, mais rien à ce stade ne m'autorise à tirer une conclusion.

16. Hauser-Schäublin (1989a: 188) rapporte la même comparaison faites par les habitants de Kalabu.

17. Cette relation explique notamment la nécessité de commencer le Yakët avant l'ouverture des essarts, la sueur produite par l'intensité du labeur de la coupe tombant sur le sol et étant alors évaluée par les agents de la pierre.

18. Également le terme utilisé pour un filet de chasse, un filet de portage, la matrice féminine et les murs des chambres initiatiques à l'intérieur de la maison cérémonielle.

19. Il existe des récits d'attaques dirigées contre des clans dont l'objectif était de détruire la pierre.

20. Il existe pourtant des exceptions. Ainsi HauserSchäublin mentionne le cas d'une pierre du village de Jaamë dont un groupe de soldats japonais, durant la Seconde Guerre Mondiale, s'empara pour l' utiliser comme pierre de foyer. Devenue inutile pour le clan, elle fut alors donnée à Kalabu qui parvint à la restaurer dans ses fonctions (Hauser-Schäublin 1989a: 186).

21. Durant mon séjour, l'essentiel de la communauté était de confession catholique, avec quelques membres associés à la New Apostolic Church.

22. L'importance du féminin et des femmes dans la culture des grandes ignames a été traitée dans le travail de Diane Losche (1982). 


\section{RÉFÉRENCES}

Aufenanger, H. 1972 The Passing scene in northeast New Guinea. Vol. 2. St. Augustin: Anthropos Institut (Collectanea Instituti Anthropos).

- 1975 The Great inheritance in northeast New Guinea. Vol. 9. St. Augustin: Anthropos Institut (Collectanea Instituti Anthropos).

Burkill, I. H. 1951 The Rise and decline of the greater yam in the service of man, Advancement of Science, 7: 443-48.

Coupaye, L. 2009 Ways of enchanting: Chaînes Opératoires and Yam Cultivation in Nyamikum Village, Maprik, Papua New Guinea, Journal of Material Culture 14 (4) : 433 - 458.

— 2010 Décrire les objets hybrides. In L. Coupaye \& L. Douny (Dir.) Technologies. Techniques \& Culture 52-53: 50-67.

— 2012 De «L'Objet social total » à la « sociologie par l'objet». L'igname comme contexte chez les Abelam de Papouasie-Nouvelle-Guinée. In A.-C. Taylor \& N. Schlanger (Dir.) Actes du colloque « La Préhistoire des Autres ». Paris: La Découverte-INRAP.

- 2013 Growing artefacts, displaying relationships: yams, art and technology amongst the Nyamikum Abelam of Papua New Guinea. Oxford \& New York: Berghahn Books.

Forge, A. 1965 Art and environment in the Sepik. Proceedings of the royal anthropological institute for $1965: 23-31$

— 1970 Learning to see in New Guinea. In P. Mayer (Dir.) Socialization: the approach from social anthropology. London, New York, Sydney, Toronto, Wellington: Tavistock: 269-91.

— 1973 Style and meaning in Sepik art. In Primitive art and society. London : Oxford University Press, Ely House: 169-192.

- 1990 The Power of culture and the culture of power. In Nancy Lutkehaus et al. (Dir.), Sepik heritage. Durham: Carolina Academic Press: 160-70.

Gell, A. 1975 Metamorphosis of the cassowaries: Umeda society, language and ritual. London: Athlone.

— 2008 [1998] L'Art et ses agents - Une théorie anthropologique. Paris: Les Presses du Réel.

Gerrits, G. J.M. (2012 [1978]) The House tambaran of Bongiora. A view from within of the tambaran and yam Cults of the Abelam in the east Sepik Province of Papua New Guinea. Lugano: Seria Antropunti, Museo delle culture Lugano et Giampiero Casagrande editore.

Godelier, M. 1996 L'Énigme du Don. Paris: Fayard.

Haudricourt, A.-G. 1987 [1962] Domesticaton des animaux, culture des plantes et traitement d'autrui. In La Technologie, science humaine, recherches d'ethnologie et d'histoire des techniques. Paris: Maison des Sciences de l'Homme: 277-285.

Hauser-Schaüblin, B. 1984 Schweinefleisch un totenseele. Zur bedeutung des schwienes in der kultur des Abelam, Papua New Guinea. Bâle: Verhandlungen der naturschenden gesellschaft in Basel, 94.

- 1995 Puberty rites, women's Naven and initiation: women's rituals of transition in Abelam and iatmul cultures's. In N. C. Lutkehaus \& P. B. Roscoe (dir.) Gender rituals: female initiations in Melanesia. New York : Routledge: 33-53.

- 1989a Kulthäuser in Nordneuguinea. Vol. 1: Architektur, funktion und symbolik des kulthauses bei den Abelam; Vol. 2: Vergleichende Studien zu Kulthaüsern im Sepik-Gebiet und an der Nordküste. Abhandlungen und berichte des staatlichen museums für volkerkunde dresden. Berlin: Akademie-Verlag.

- 1989b Leben in linie, muster und farbe: einführung in die betrachtung aussereuropaïscher kunst am beispiel der Abelam, Papua-Neuguinea. Basel: Birkhäuser Verlag.

Heermann, I. 1983 Ein Lingwallndu-initiations-raum der Abelam: die sammlung gerrits im linden-Museum Stuttgart. Tribus 32: 127-169.

Huber-Greub, B. 1988 Kokospalmenmenschen: boden und alltag und ihre bedeutung im selbstverständnis der Abelam von kimbangwa (east Sepik province, Papua New Guinea). Basler Beiträger zur Ethnologie, Band 27. Basel: Weipf \& Co. AG Verlag. 
Kaberry, P. 1941 The Abelam tribe, Sepik District, New Guinea: a preliminary report. Oceania 11 (3) : 233258; (4): 345-367.

Kaeppler, A, Kaufmann, C. \& Newton, D. 1993 L’Art océanien. Paris: Citadelle \& Mazenod.

Kaufmann, C. 1993 La Mélanésie. In A. Kaeppler, C. Kaufmann et D. Newton. L’Art océanien. Paris: Citadelle \& Mazenod: 159-403.

— 2005 Canon et style. Diversité stylistique dans l'art Kwoma de Papouasie Nouvelle Guinée. In B. Martinelli (dir.) L'Interrogation du style. Anthropologie, technique et esthétique. Marseille: Publications de l'université de Provence.

Küchler, S. 1992 Making skins: malangan and the idiom of kinship in northern New Ireland. In J. Coote \& A. Shelton (dir.) Anthropology art and aesthetics. Oxford :Clarendon Press, 94-112.

Kudama, J. \& Wilson, P. 1987 Kudi kupuk: ambulas, tok pisin, english. Dictionaries of Papua New Guinea, volume 10. Ukarumpa, Papua New Guinea: Summer Institute of Linguistics.

Lea, D. A. M. 1964 Abelam land and sustenance horticulture in an area of high population density, Maprik, New Guinea. Australian National University: Canberra (Thèse)

— 1966 Yam growing in the Maprik Area. Papua New Guinea Agricultural Journal 18 (1) : 5-15.

Lemonnier, P. 2012 Des Objets pour penser l'indicible: la nécessaire convergence des théories de la culture matérielle». In A.-C. Taylor et N. Schlanger (dir.) Actes du colloque La Préhistoire des Autres. Paris: La Découverte-INRAP.

Losche, D. 1982 Male and female in Abelam society. Ann Arbor, Michigan: University Microfilms International. New York: Columbia University (Thèse).

Mauss, M. 1950 (1935) Les Techniques du corps. In Sociologie et anthropologie. Paris: PUF: 365-386.

McGuigan, N. 1992 The Social context of Abelam art: a comparison of art, religion and Leadership in Two Abelam Communities. Belfast: the University of Ulster (Thèse soumise pour le diplôme de Doctor of Philosophy).

Morphy, H. 2005 Style and meaning: abelam art through Yolngu eyes. Res, 47: 209-230.

Revolon, S. 2007 Les esprits aiment ce qui est beau. Formes, sens et efficacité rituelle des sculptures owa (est des îles Salomon). Annales de la Fondation Fyssen, 21: 63-73.

Smidt, D. \& McGuigan N. 1994 An Emic and etic role for Abelam art (Papua New Guinea): the context of a collecting trip on behalf of the Rijksmuseum voor Volkenkunde, Leiden. In P. C. Dark \& R. Rose (dir.) Artistic heritage in a changing pacific. Honolulu: University of Hawaii Press: 121-141.

Thurnwald, R. C. 1914 Vom mittleren Sepik zur nordwestküste von Kaiser-Wilhelmsland. Mitteilungen aus den deutschen schutzgebieten 27 (1): 81-84.

Tuzin, D. 1972 Yam symbolism in the Sepik: an interpretation account. Southwestern Journal of Anthropology XXVIII: 230-254.

— 1976 The Voice of the tambaran: truth and illusion in Ilahita arapesh religion. Berkeley, Los Angeles \& London: University of California Press.

- 1995 Art and procreative illusion in the Sepik: comparing the Abelam and the Arapesh. Oceania 65 (4): 289-303.

- 2002 Arts, ritual, and the crafting of illusion. The Asia Pacific Journal of Anthropology 3 (1): 1-23.

Weiner, A. B. 1992 Inalieneable possessions: the Paradox of keeping-while-giving. Berkeley : University of California Press. 


\section{RÉSUMÉ}

Des Images, des nouds et des toiles. Pierres sacrées et ignames à Nyamikum (district de maprik, East Sepik province, Papouasie-Nouvelle Guinée). La notion d'objet irremplaçable peut s'appliquer à la fois aux objets considérés comme tels par les sociétés que les anthropologues étudient, mais aussi pour ces derniers qui cherchent à décrire des pratiques, des institutions ou des représentations. Ces trois phénomènes ne se manifestent jamais sur le terrain de manière totalement intangible mais toujours en relation avec des objets. Ainsi, les pierres sacrées des Abelam de Papouasie-Nouvelle-Guinée sont des objets qui, bien qu'invisibles parce que secrets, sont essentielles pour la culture et les rituels d'exposition des grandes ignames décorées (Coupaye 2010), célèbres dans la littérature ethnographique. Ces pierres sont également «irremplaçables » pour notre compréhension d'un phénomène souvent interprété comme un culte phallique masculin, mais qui touche plus largement à l'ancrage de l'identité des groupes dans le temps et le territoire, incluant de nombreuses espèces végétales, animales et des esprits. Les pratiques qui entourent ces pierres sacrées nous permettent alors d'affiner nos interprétations et d'y intégrer des éléments généralement abordés de manière dispersée, tels que les relations sociales entre les clans, les villages, les mythes, les rites, les espèces naturelles et les entités surnaturelles.

\section{ABSTRACT}

Images, nodes and networks. The sacred stones of the yams of Nyamikum (district of maprik, East Sepik province, apua new Guinea). The notion of irreplaceable objects can be applied both to object considered as such by the societies studied by anthropologists, as well as by the latter who want to describe practices, insitutions or representations. These three phenomena are never totally intangible and are always in relations with objects or artefacts. The sacred stones of the Abelam of Papua new Guinea are objects which, though invisible and secret, are essential for the cultivation and exhibition of decorated yams (Coupaye 2010). These stones are also essential for our understanding of a phenomenon, often considered only as a male phallic cult, but which in fact concerns more widely the temporal and territorial anchoring of human groups, in relation with many vegetal and animal species, as well as with spiritual beings. The practices surrounding these sacred stones allow us to sophisticate our interpretations by including within them social relations between clans, villages, myths, rituals, natural species and surnatural entities.

\section{MOTS CLÉS}

Culture matérielle, relations sociales, culture (jardins), pierres sacrées, rituels, mythes.

\section{KEYWORDS}

Material culture, social relations, garden cultivation, sacred stones, rituals, myths. 